\title{
Targeting metastatic colorectal cancer - present and emerging treatment options
}

This article was published in the following Dove Press journal:

Pharmacogenomics and Personalized Medicine

8 July 2014

Number of times this article has been viewed

\author{
Kristen K Ciombor \\ Jordan Berlin² \\ 'Division of Medical Oncology, \\ Department of Medicine, The Ohio \\ State University Comprehensive \\ Cancer Center, Columbus, OH, USA \\ ${ }^{2}$ Division of Hematology/Oncology, \\ Department of Medicine, Vanderbilt- \\ Ingram Cancer Center, Nashville, \\ TN, USA
}

\begin{abstract}
Metastatic colorectal cancer is a significant cause of morbidity and mortality in the US and around the world. While several novel cytotoxic and biologic therapies have been developed and proven efficacious in the past two decades, their optimal use in terms of patient selection, drug combinations, and regimen sequences has yet to be defined. Recent investigations regarding anti-epidermal growth factor receptor therapies include the comparison of single-agent panitumumab and cetuximab, the benefit of adding cetuximab to chemotherapy in the conversion therapy setting, the comparison of cetuximab and bevacizumab when added to first-line chemotherapy, and predictive biomarkers beyond KRAS exon 2 (codons 12 and 13) mutations. With respect to anti-vascular endothelial growth factor therapies, new data on continuing bevacizumab beyond disease progression on a bevacizumab-containing chemotherapy regimen, the addition of bevacizumab to triplet chemotherapy in the first-line setting, maintenance therapy with bevacizumab plus either capecitabine or erlotinib, the addition of aflibercept to chemotherapy, and regorafenib as monotherapy have emerged. Recent scientific and technologic advances in the field of metastatic colorectal cancer promise to elucidate the biological underpinnings of this disease and its therapies for the goal of improving personalized treatments for patients with metastatic colorectal cancer.
\end{abstract}

Keywords: cetuximab, panitumumab, bevacizumab, aflibercept, regorafenib, biomarker

\section{Introduction}

Colorectal cancer (CRC) is the third leading cause of cancer-related death in the US, and third in cancer prevalence in both men and women. An estimated 142,820 new cases and 50,830 deaths from CRC are expected in 2013 alone. ${ }^{1}$ While important efforts in the prevention and early detection of CRC are ongoing, approximately one-fifth of patients diagnosed with CRC will have evidence of distant spread at diagnosis. ${ }^{1}$ Advances in systemic chemotherapeutics have led to a remarkable improvement in overall survival (OS) for patients with metastatic CRC (mCRC) since the era of singleagent 5-fluorouracil (5-FU). Notably, the incorporation of oxaliplatin, irinotecan, bevacizumab, cetuximab, panitumumab, aflibercept, and regorafenib into treatment strategies for mCRC beyond 5-FU has easily doubled the median OS for this disease, with more patients enjoying long-term survival (Table 1). As we advance our knowledge of the underlying biology of $\mathrm{mCRC}$, appropriate development and use of targeted therapies such as anti-epidermal growth factor receptor (EGFR) and anti-vascular endothelial growth factor (VEGF) agents and others promise to further improve our treatment of this prevalent disease.
Correspondence: Jordan Berlin Division of Hematology/Oncology, Department of Medicine, VanderbiltIngram Cancer Center, 777 Preston Research Building, 2220 Pierce Avenue, Nashville, TN 37232, USA

$\mathrm{Tel}+\mathrm{I} 6153224967$

Fax + I 6153437602

Email jordan.berlin@vanderbilt.edu 
Table I Selected Phase III trials employing targeted biologic therapies with or without chemotherapy in metastatic colorectal cancer

\begin{tabular}{|c|c|c|c|c|}
\hline Trial & Biologic \pm chemotherapy & Sample size & Endpoints & $P$-value \\
\hline $\begin{array}{l}\text { Price et al }{ }^{17} \\
\text { ASPECCT }\end{array}$ & $\begin{array}{l}\text { Cetuximab vs panitumumab } \\
\text { in refractory } \mathrm{mCRC}\end{array}$ & $\mathrm{N}=999$ & $\begin{array}{l}\text { OS: } 10.0 \text { vs } 10.4 \text { mos } \\
\text { PFS: } 4.4 \text { vs } 4.1 \text { mos } \\
\text { ORR: } 19.8 \% \text { vs } 22 \%\end{array}$ & $P=0.0007$ \\
\hline Ye et al ${ }^{18}$ & $\begin{array}{l}\text { Chemotherapy (mFOLFOX } 6 \text { or } \\
\text { FOLFIRI) } \pm \text { cetuximab in first-line } \\
\text { mCRC therapy }\end{array}$ & $N=138$ & $\begin{array}{l}\text { R0 resection rate for liver mets: } \\
25.7 \% \text { vs } 7.4 \% \\
\text { ORR: } 57.1 \% \text { vs } 29.4 \% \\
\text { OS: } 30.9 \text { vs } 21.0 \text { mos }\end{array}$ & $\begin{array}{l}P<0.01 \\
P<0.01 \\
P=0.013\end{array}$ \\
\hline $\begin{array}{l}\text { Heinemann et al }{ }^{19} \\
\text { FIRE-3 } \\
\text { (AIO KRK-0306) }\end{array}$ & $\begin{array}{l}\text { FOLFIRI + cetuximab vs FOLFIRI + } \\
\text { bevacizumab in first-line } \mathrm{mCRC} \\
\text { therapy }\end{array}$ & $\mathrm{N}=508$ & $\begin{array}{l}\text { ORR: } 62 \% \text { vs } 58 \% \\
\text { PFS: } 10.0 \text { vs } 10.3 \text { mos } \\
\text { OS: } 28.7 \text { vs } 25 \text { mos }\end{array}$ & $\begin{array}{l}P=0.183 \\
P=0.547 \\
P=0.017\end{array}$ \\
\hline $\begin{array}{l}\text { Bennouna et } \mathrm{al}^{32} \\
\text { MLI } 8 \mid 47\end{array}$ & $\begin{array}{l}\text { Second-line chemotherapy } \pm \\
\text { bevacizumab }\end{array}$ & $\mathrm{N}=820$ & OS: 11.2 vs $9.8 \mathrm{mos}$ & $P=0.0062$ \\
\hline $\begin{array}{l}\text { Falcone et } \mathrm{al}^{36} \\
\text { TRIBE }\end{array}$ & $\begin{array}{l}\text { FOLFOXIRI + bevacizumab vs } \\
\text { FOLFIRI + bevacizumab }\end{array}$ & $\mathrm{N}=508$ & $\begin{array}{l}\text { PFS: } 12.2 \text { vs } 9.7 \text { mos } \\
\text { ORR: } 65 \% \text { vs } 53 \% \\
\text { R0 resection rate: I5\% vs I } 2 \% \\
\text { OS: } 31.0 \text { vs } 25.8 \text { mos }\end{array}$ & $\begin{array}{l}P=0.0012 \\
P=0.006 \\
P=0.327 \\
P=0.054\end{array}$ \\
\hline $\begin{array}{l}\text { Punt et al }{ }^{39} \\
\text { CAIRO-3 }\end{array}$ & $\begin{array}{l}\text { CAPOX-B followed by maintenance } \\
\text { capecitabine }+ \text { bevacizumab } \\
\text { or observation }\end{array}$ & $\mathrm{N}=558$ & $\begin{array}{l}\text { PFSI: } 8.5 \text { vs } 4.1 \text { mos } \\
\text { PFS2: } 11.5 \text { vs } 10.5 \text { mos } \\
\text { TTP2: } 18.7 \text { vs } 14.1 \text { mos } \\
\text { OS: } 21.7 \text { vs } 18.0 \text { mos }\end{array}$ & $\begin{array}{l}P<0.0001 \\
P=0.03 \\
P<0.0001 \\
P=0.16\end{array}$ \\
\hline $\begin{array}{l}\text { Johnsson et al }{ }^{40} \\
\text { Nordic ACT }\end{array}$ & $\begin{array}{l}\text { First-line doublet chemotherapy }+ \\
\text { bevacizumab, followed by } \\
\text { bevacizumab } \pm \text { erlotinib }\end{array}$ & $\mathrm{N}=249$ & $\begin{array}{l}\text { PFS: } 5.7 \text { vs } 4.2 \mathrm{mos} \\
\text { OS: } 21.5 \text { vs } 22.8 \mathrm{mos}\end{array}$ & $\begin{array}{l}P=0.19 \\
P=0.51\end{array}$ \\
\hline $\begin{array}{l}\text { Van Cutsem et al }{ }^{42} \\
\text { VELOUR }\end{array}$ & $\begin{array}{l}\text { FOLFIRI + aflibercept vs FOLFIRI + } \\
\text { placebo in previously oxaliplatin- } \\
\text { treated mCRC }\end{array}$ & $\mathrm{N}=1,226$ & $\begin{array}{l}\text { OS: } 13.50 \text { vs } 12.06 \text { mos } \\
\text { ORR: } 19.8 \% \text { vs } 11.1 \% \\
\text { PFS: } 6.90 \text { vs } 4.67 \mathrm{mos}\end{array}$ & $\begin{array}{l}P=0.0032 \\
P=0.0001 \\
P<0.0001\end{array}$ \\
\hline $\begin{array}{l}\text { Grothey et al }{ }^{45} \\
\text { CORRECT }\end{array}$ & $\begin{array}{l}\text { Regorafenib vs placebo } \\
\text { in refractory } \mathrm{mCRC}\end{array}$ & $N=I, 5 \mid 3$ & $\begin{array}{l}\text { OS: } 6.4 \text { vs } 5.0 \text { mos } \\
\text { PFS: } 1.9 \text { vs } 1.7 \text { mos } \\
\text { ORR: } 1.0 \% \text { vs } 0.4 \%\end{array}$ & $\begin{array}{l}P=0.0052 \\
P<0.0001 \\
P=0.19\end{array}$ \\
\hline
\end{tabular}

Abbreviations: mCRC, metastatic colorectal cancer; mets, metastases; mos, months; ORR, overall response rate; OS, overall survival; PFS, progression-free survival; PFSI, PFS at first progression; PFS2, PFS at second progression; TTP2, time to second progression; FOLFOX, chemotherapy regimen involving drugs folinic acid, fluorouracil, and oxaliplatin; FOLFIRI, chemotherapy regimen involving drugs folinic acid, fluorouracil, and irinotecan; FOLFOXIRI, chemotherapy regimen involving folinic acid, fluorouracil, oxaliplatin and irinotecan; CAPOX-B, chemotherapy regimen involving capecitabine, oxaliplatin and bevacizumab.

\section{CRC and its underlying biology: implications for therapy}

The development of CRC occurs as a result of specific genetic alterations, many of which have been elucidated or further clarified with recent advances in molecular technologies. The well-known Vogelstein model has long hypothesized that germline or somatic mutations are required for malignant transformation, and the accumulation of multiple mutations determines the biological behavior of the tumor. ${ }^{2}$ Furthermore, three molecular pathways have been implicated in colorectal tumorigenesis, and these include the chromosomal instability pathway, ${ }^{3}$ the mutator-phenotype/ DNA mismatch repair pathway, ${ }^{4}$ and the hypermethylation phenotype, or hyperplastic/serrated polyp pathway. ${ }^{5}$ While a detailed description of these pathways is outside the scope of this article, it is important to recognize that distinctive molecular characteristics of these pathways have implications for targeted therapies and their potential efficacy. For example, mutator-phenotype tumors from patients with hereditary nonpolyposis CRC (HNPCC) only demonstrate KRAS mutations and not $B R A F$ mutations, whereas $B R A F$ mutations are seen almost entirely in hypermethylation pathway tumors that are $K R A S$ wild-type. ${ }^{6,7}$ Understanding the underlying biology of how colorectal tumors form is therefore critically important in developing effective personalized therapies for patients with this disease.

\section{Anti-EGFR treatment strategies: controversies regarding optimal sequence and use}

The anti-EGFR monoclonal antibodies $(\mathrm{mAb})$ cetuximab (an immunoglobin [Ig]G1 chimeric $\mathrm{mAb}$ ) and panitumumab (a fully human $\mathrm{mAb}$ ) have proven efficacy in the treatment of $K R A S$ wild-type $\mathrm{mCRC}$, both as monotherapy and in combination with chemotherapy in various settings (Table 2). ${ }^{8-16}$ However, their optimal use and sequence in therapy is not defined. 
Table 2 Summary of approved targeted therapies for metastatic colorectal cancer

\begin{tabular}{|c|c|c|c|c|}
\hline Drug & Class & $\begin{array}{l}\text { Method of } \\
\text { administration }\end{array}$ & Target(s) & Pathway(s) \\
\hline Cetuximab (Erbitux ${ }^{\circledR}$ ) & Monoclonal antibody (IgGI chimeric) & Intravenous & EGFR & PI3K/Akt, MAPK \\
\hline Panitumumab (Vectibix ${ }^{\circledR}$ ) & Monoclonal antibody (fully human) & Intravenous & EGFR & PI3K/Akt, MAPK \\
\hline Bevacizumab (Avastin $\left.{ }^{\circledR}\right)$ & Monoclonal antibody & Intravenous & VEGF-A & Angiogenesis \\
\hline Aflibercept (Zaltrap $\left.{ }^{\circledR}\right)$ & Recombinant fusion protein & Intravenous & $\begin{array}{l}\text { VEGF-A, VEGF-B, } \\
\text { PIGF-I, PIGF-2 }\end{array}$ & Angiogenesis \\
\hline Regorafenib (Stivarga ${ }^{\circledR}$ ) & Tyrosine kinase inhibitor & Oral & $\begin{array}{l}\text { VEGFRI, VEGFR2, VEGFR3, } \\
\text { PDGFR- } \beta \text {, FGFRI, TIE2, } \\
\text { KIT, RET, BRAF }\end{array}$ & $\begin{array}{l}\text { Angiogenesis, } \\
\text { oncogenesis, tumor } \\
\text { microenvironment }\end{array}$ \\
\hline
\end{tabular}

Notes: Manufacturers are as follows: Erbitux: Bristol-Myers Squibb, New York, NY, USA; Vectibix: Amgen Inc., Thousand Oaks, CA, USA; Avastin: Genentech, Inc., South San Francisco, CA, USA; Zaltrap: Sanofi-Aventis, Bridgewater, NJ, USA; Stivarga: Bayer AG, Leverkusen, Germany.

Abbreviations: EGFR, epidermal growth factor receptor; FGFR, fibroblast growth factor receptor; Ig, immunoglobulin; MAPK, mitogen-activated protein kinase; PDGFR, platelet-derived growth factor receptor; PI3K, phosphatidylinositide 3-kinase; PIGF, placental growth factor; VEGF, vascular endothelial growth factor; VEGFR, vascular endothelial growth factor receptor.

Important questions regarding their use that have been recently investigated include the comparable efficacy of cetuximab versus panitumumab, as well as anti-EGFR therapy versus other biologics, and potential biomarkers to more accurately predict for response to anti-EGFR therapy.

\section{Single-agent cetuximab versus panitumumab in the refractory mCRC setting}

As single agents, both cetuximab and panitumumab have efficacy in the treatment of patients with $K R A S$ wild-type mCRC. It was unclear, however, whether cetuximab had a survival advantage over panitumumab in the refractory setting. Therefore, the non-inferiority, Phase III ASPECCT trial sought to compare the efficacy and safety of cetuximab and panitumumab in chemorefractory mCRC (Table 1$).{ }^{17} \mathrm{~A}$ total of 999 mCRC patients previously treated with irinotecan, oxaliplatin, and fluorouracil-based treatment were randomized $1: 1$ to either single-agent cetuximab $\left(400 \mathrm{mg} / \mathrm{m}^{2}\right.$ intravenous [IV] loading dose, followed by $250 \mathrm{mg} / \mathrm{m}^{2} \mathrm{IV}$ weekly) or panitumumab (6 mg/kg IV every 2 weeks). Non-inferiority in this trial was defined as panitumumab preserving at least $50 \%$ of the cetuximab OS effect when compared to best supportive care. With a median OS of 10.4 versus 10.0 months for panitumumab and cetuximab, respectively, the non-inferiority endpoint was met (hazard ratio [HR]: 0.97, 95\% confidence interval [CI]: 0.84-1.11, P=0.0007). Additionally, median progression-free survival (PFS; 4.1 versus [vs] 4.4 months for panitumumab vs cetuximab, HR: 1.00, 95\% CI: 0.88-1.14) and overall response rates (ORR) (22\% vs $19.8 \%$ for panitumumab vs cetuximab) were similar. Given the similar results of the two agents in this trial, it was concluded that these therapies have equivalent efficacy as monotherapy for $\mathrm{mCRC}$.

\section{Anti-EGFR therapy in combination with first-line chemotherapy as conversion therapy}

Given the established efficacy of cetuximab in combination with chemotherapy in the first- and second-line settings, Chinese investigators sought to determine whether the addition of cetuximab to first-line chemotherapy could increase the rate of metastasectomy in patients with liver-limited, unresectable, KRAS wild-type mCRC (Table 1). ${ }^{18}$ A total of 138 patients were randomly assigned to mFOLFOX6 (folinic acid, 5-FU, and oxaliplatin) or FOLFIRI (folinic acid, 5-FU, and irinotecan) plus cetuximab (loading dose of $400 \mathrm{mg} / \mathrm{m}^{2}$ followed by $250 \mathrm{mg} / \mathrm{m}^{2}$ weekly, or $500 \mathrm{mg} / \mathrm{m}^{2}$ on day 1 and every 2 weeks thereafter) or chemotherapy alone. Patients on the cetuximab-containing arm were found to have improved R0 resection rates for liver metastases $(25.7 \%$ vs $7.4 \%, P<0.01)$, objective response rates $(57.1 \%$ vs $29.4 \%$, $P<0.01$ ), and median survival time (30.9 vs 21.0 months, $P=0.013)$. These results add to those of prior studies suggesting that the addition of an anti-EGFR agent to chemotherapy may be important in improving outcomes, including OS, with conversion therapy.

\section{Anti-EGFR versus anti-VEGF therapy in combination with first-line chemotherapy}

To investigate the potential superiority of anti-VEGF or antiEGFR therapy in combination with first-line chemotherapy, the Phase III FIRE-3 (AIO KRK-0306) trial randomized patients with $K R A S$ wild-type (exon 2) mCRC to treatment with FOLFIRI at standard doses plus cetuximab $\left(400 \mathrm{mg} / \mathrm{m}^{2}\right.$ on day 1 , followed by $250 \mathrm{mg} / \mathrm{m}^{2}$ weekly) or bevacizumab ( $5 \mathrm{mg} / \mathrm{kg}$ every 2 weeks; Table 1$).{ }^{19}$ This study did not meet its primary endpoint of improvement in ORR, as ORR was comparable between arms ( $62 \%$ vs $58 \%$ for the cetuximab- vs 
bevacizumab-containing arms, respectively; odds ratio (OR): 1.18, 95\% CI: $0.85-1.64, P=0.183)$. PFS between the two arms was similar as well (10.0 vs 10.3 months, HR: 1.06, 95\% CI: 0.88-1.26, $P=0.547)$. Interestingly, however, OS was significantly improved in the cetuximab-containing arm (28.7 vs 25 months, HR: 0.77, 95\% CI: 0.62-0.96, $P=0.017$ ). The presence of a benefit in OS but lack thereof in PFS and ORR for the cetuximab-containing arm is puzzling, but it may be at least partially explained by subsequent therapies that the study patients received. Results from a completed intergroup study comparing first-line treatment with either FOLFOX or FOLFIRI and cetuximab and/or bevacizumab, CALGB 80405, are expected in 2014 and should shed additional light on this subject. ${ }^{20,21}$ In the meantime, however, the FIRE-3 results underscore the need for predictive biomarkers to more accurately select patients appropriate for anti-EGFR and/or anti-VEGF therapies.

\section{Exon 2 KRAS wild-type status as a predictive biomarker for anti-EGFR therapy: necessary but not sufficient}

While it is known that patients with $K R A S$ mutations in codons 12 and 13 (exon 2) do not benefit from the anti-EGFR agents cetuximab and panitumumab, it was unclear whether other $R A S$ mutations were negative predictive biomarkers for anti-EGFR therapy as well. A mutational analysis of the Panitumumab Randomized Trial in Combination with Chemotherapy for Metastatic Colorectal Cancer to Determine Efficacy (PRIME) study, in which mCRC patients were randomized to FOLFOX4 with or without panitumumab $6 \mathrm{mg} / \mathrm{kg}$ IV every 2 weeks, was therefore performed. ${ }^{22}$ Tumor tissue samples were analyzed for mutations in $K R A S$ exons 3 (codon 61) and 4 (codons 117, 146); NRAS exons 2 (codons 12, 13), 3 (codon 61), and 4 (codons 117, 146); and $B R A F$ exon 15 (codon 600). Patients without any $R A S$ mutations had improved PFS (10.1 vs 7.9 months, HR: 0.72 , 95\% CI: $0.58-0.90, P=0.0004)$ and OS (26.0 vs 20.2 months, HR: $0.78,95 \% \mathrm{CI}$ : 0.62-0.99, $P=0.04)$ when panitumumab was added to FOLFOX4 chemotherapy. Additionally, the presence of any $R A S$ mutation was associated with inferior PFS and OS with panitumumab-FOLFOX4 treatment.

Similar to the PRIME study, mutational status beyond exon 2 of $K R A S$ was found to be important in the FIRE-3 trial as well. When the FIRE-3 patients' tumors were tested for $K R A S$ exons 3 (codons 59, 61) and 4 (codons 117, 146); NRAS exons 2 (codons 12, 13), 3 (codons 59, 61), and 4 (codons 117, 146); and $B R A F$ (V600E), the wild-type $R A S$ group demonstrated an increased ORR when treated with FOLFIRI/cetuximab
(76.0\% vs $65.2 \%$, Fisher's two-sided $P=0.044) .{ }^{23}$ PFS was comparable in the wild-type $R A S$ group treated with cetuximab versus bevacizumab (10.5 vs 10.4 months, HR: 0.94, 95\% CI: $0.75-1.19, P=0.63)$, but OS was significantly prolonged with cetuximab (33.1 vs 25.9 months, HR: 0.69, 95\% CI: $0.52-0.92, P=0.01)$. Though retrospective, the PRIME and FIRE-3 data emphasize the importance of continued predictive biomarker investigation and discovery based on underlying biologic mechanisms of mCRC.

\section{Anti-VEGF treatment strategies: controversies regarding optimal choice of agent and sequence}

Anti-VEGF therapy in the form of bevacizumab (Avastin ${ }^{\circledR}$; Genentech, Inc., South San Francisco, CA, USA), a monoclonal antibody that targets VEGF-A, has been an important adjunct in the treatment of mCRC for almost a decade (Table 2). Investigations regarding its use, particularly in the second-line setting after progression with bevacizumab-containing chemotherapy, and in combination with capecitabine or erlotinib in the maintenance setting, have been undertaken and recently published. ${ }^{32,38-40}$ Additionally, agents with antiangiogenic effects such as aflibercept (Zaltrap ${ }^{\circledR}$; Sanofi-Aventis, Bridgewater, NJ, USA) and regorafenib (Stivarga ${ }^{\circledR}$; Bayer AG, Leverkusen, Germany) have been developed in attempts to maximize anti-VEGF targeting (Table 2). Questions remain regarding optimal treatment settings in which to employ these agents, as well as biomarkers to more accurately predict response to therapy.

\section{Bevacizumab beyond progression}

Bevacizumab has proven clinical efficacy when used in combination with first- or second-line fluorouracil-based chemotherapy. ${ }^{24-29}$ Given this efficacy, it was wondered whether bevacizumab was beneficial when continued beyond disease progression on first-line, bevacizumab-containing chemotherapy. Observational studies had previously hypothesized a benefit to bevacizumab beyond progression, ${ }^{30,31}$ but recent prospective data confirmed the benefit. In the Phase III ML18147 trial, 820 patients with $\mathrm{mCRC}$ with progressive disease after first-line bevacizumab plus chemotherapy were randomized to second-line chemotherapy with or without bevacizumab (2.5 mg/kg IV per week equivalent; Table 1). ${ }^{32}$ There was an OS benefit to continuing bevacizumab with chemotherapy in the second-line setting when compared to chemotherapy alone (median OS: 11.2 vs 9.8 months, HR: 0.81, 95\% CI: 0.69-0.94, unstratified log-rank tested $P=0.0062$ ). As a result, maintaining angiogenesis inhibition 
through the continued use of bevacizumab while switching chemotherapies after disease progression on first-line regimens has become a standard in the treatment of $\mathrm{mCRC}$ patients.

\section{Bevacizumab plus triplet chemotherapy}

Given the efficacy of doublet fluorouracil-based regimens for mCRC (ie, FOLFOX, FOLFIRI), it was wondered whether the combination of 5-FU, irinotecan, and oxaliplatin in the first-line setting would be more efficacious than doublet therapy. The triplet regimen FOLFOXIRI (folinic acid, 5-FU, oxaliplatin and irinotecan) has been studied in two Phase III trials with encouraging results, although sample sizes were small and chemotherapy doses not consistent. ${ }^{33-35}$ Given that bevacizumab is relatively well-tolerated in patients without contraindications to its therapy and that it is a standard in combination with doublet chemotherapy, investigators hypothesized that the addition of bevacizumab to triplet chemotherapy may further increase its benefit. The Phase III TRIBE trial randomized 508 untreated mCRC patients to either FOLFOXIRI/bevacizumab (irinotecan $165 \mathrm{mg} / \mathrm{m}^{2}$, oxaliplatin $85 \mathrm{mg} / \mathrm{m}^{2}$, leucovorin $200 \mathrm{mg} / \mathrm{m}^{2}, 5-\mathrm{FU}$ infusion of $3,200 \mathrm{mg} / \mathrm{m}^{2}$ over 48 hours, and bevacizumab $5 \mathrm{mg} / \mathrm{kg}$ ) or FOLFIRI/bevacizumab (irinotecan $180 \mathrm{mg} / \mathrm{m}^{2}$, leucovorin $200 \mathrm{mg} / \mathrm{m}^{2}, 5-\mathrm{FU}$ bolus $400 \mathrm{mg} / \mathrm{m}^{2}$, followed by $5-\mathrm{FU}$ infusion of $2,400 \mathrm{mg} / \mathrm{m}^{2}$ over 48 hours, and bevacizumab $5 \mathrm{mg} / \mathrm{kg}$ ) in order to answer this question (Table 1). ${ }^{36,37}$ Of note, therapy was continued for up to 12 cycles and then followed by 5 -FU/bevacizumab until disease progression. In this study, treatment with FOLFOXIRI/bevacizumab led to improved median PFS (12.2 vs 9.7 months, HR: 0.73, 95\% CI: $0.60-0.88, P=0.0012)$ and response rate $(65 \%$ vs $53 \%$, $P=0.006)$ when compared to FOLFIRI/bevacizumab, but did not increase the $\mathrm{R} 0$ secondary resection rate $(15 \%$ vs $12 \%, P=0.327$ ) or median OS (31.0 vs 25.8 months, HR: $0.79, P=0.054)$. Not unexpectedly, toxicities were greater in the FOLFOXIRI/bevacizumab arm. While these data are not encouraging enough to adopt FOLFOXIRI/bevacizumab as the standard of care in first-line chemotherapy, it can be considered for selected patients.

\section{Maintenance anti-VEGF and anti-EGFR therapy in $\mathrm{mCRC}$}

Another key question with respect to biologic therapy in mCRC involves the use of maintenance anti-VEGF therapy with or without anti-EGFR therapy. The CAIRO-3 study from the Dutch Colorectal Cancer Group (DCCG) sought to investigate the efficacy of maintenance bevacizumab in
mCRC patients who had not progressed after six cycles of induction treatment with capecitabine, oxaliplatin, and bevacizumab (CAPOX-B; Table 1) ${ }^{38,39}$ A total of 558 patients were randomized to either observation or maintenance treatment with lower dose, continuous capecitabine $625 \mathrm{mg} / \mathrm{m}^{2}$ orally twice daily, and bevacizumab $7.5 \mathrm{mg} / \mathrm{kg}$ IV every 3 weeks. At first progression (PFS1), patients in both arms were treated with CAPOX-B until second progression (PFS2). Recent updated analysis of this trial demonstrated an improvement in median PFS1 with capecitabine/bevacizumab treatment ( 8.5 vs 4.1 months, HR: $0.44,95 \%$ CI: $0.37-0.53, P<0.0001)$ and a small but statistically significant improvement in median PFS2 (11.5 vs 10.5 months, HR: 0.81, 95\% CI: 0.67-0.98, $P=0.03)$, despite a smaller percentage of the maintenance therapy arm receiving CAPOX-B at PFS1 (47\% vs 75\%). ${ }^{39}$ Median time to second progression (TTP2) was improved with maintenance capecitabine/bevacizumab (18.7 vs 14.1 months, HR: 0.67, 95\% CI: 0.56-0.82, $P<0.0001)$, but OS was not (21.7 vs 18.0 months, HR: 0.87, 95\% CI: $0.71-1.06, P=0.16)$. Overall quality of life was not different between the two arms.

To test the hypothesis that combined anti-VEGF and antiEGFR therapy would be superior to single-agent biologic maintenance therapy, the Phase III Nordic ACT trial was performed (Table 1). In this study, 249 mCRC patients received first-line doublet chemotherapy (XELOX, XELIRI, FOLFOX, or FOLFIRI) and bevacizumab for 18 weeks. ${ }^{40}$ Those with at least stable disease at the completion of induction therapy were then randomized to bevacizumab $7.5 \mathrm{mg} / \mathrm{kg}$ IV every 3 weeks with or without erlotinib $150 \mathrm{mg}$ daily, an anti-EGFR tyrosine kinase inhibitor. Median PFS was similar between arms (5.7 months for the combination arm vs 4.2 months for bevacizumab alone, HR: $0.79,95 \% \mathrm{CI}: 0.55-1.12, P=0.19)$, as was median OS from the start of maintenance treatment (21.5 vs 22.8 months, HR: $0.88,95 \%$ CI: $0.61-1.27, P=0.51$ ). As expected, higher rates of grades 3 and 4 adverse events were seen with the combination arm (53\% vs $13 \%)$. As a result, combined anti-VEGF and anti-EGFR maintenance therapy is not a standard at this time.

\section{Aflibercept: a decoy VEGF receptor}

While bevacizumab has proven efficacy in the treatment of mCRC, other agents with antiangiogenic properties have been investigated with the goal of more complete antiangiogenic inhibition. Aflibercept, a recombinant fusion protein containing VEGF-binding portions from the extracellular domains of human VEGF receptors 1 and 2, fused to the Fc portion of human IgG1, is an example of one such agent. ${ }^{41}$ Aflibercept 
acts as a decoy VEGF receptor, preventing ligands such as VEGF-A, VEGF-B, and placental growth factor (P1GF)-1 and PlGF-2 from binding to their endogenous receptors. After promising early phase clinical trials with aflibercept, the Phase III VELOUR trial randomized 1,226 mCRC patients previously treated with oxaliplatin-based chemotherapy to FOLFIRI plus placebo or FOLFIRI plus aflibercept $4 \mathrm{mg}$ / kg IV every 2 weeks (Table 1). ${ }^{42}$ The addition of aflibercept to FOLFIRI improved OS when compared to the addition of placebo to FOLFIRI (median OS 13.50 vs 12.06 months, HR: $0.817,95 \%$ CI: $0.713-0.937, P=0.0032)$. ORR was significantly increased with aflibercept $(19.8 \%$ vs $11.1 \%$, 95\% CI: $8.5 \%-13.8 \%, P=0.0001$ ), as was PFS (median PFS 6.90 vs 4.67 months, HR: 0.758, 95\% CI: 0.661-0.869, $P<0.0001)$.

Interestingly, this survival benefit was seen even in the $30 \%$ of patients who had previously received bevacizumab. This suggests either that aflibercept's benefit is mechanistically different from bevacizumab, or that the benefit to agents with antiangiogenic properties beyond disease progression in the first-line setting is perhaps not limited to bevacizumab. In any case, the survival benefit of aflibercept is small, and similar benefit has not been seen in other mCRC treatment settings, either as monotherapy or in combination with other chemotherapeutic agents. Furthermore, predictive biomarkers for aflibercept have not yet been discovered, though there is a suggestion that patients with liver-only metastases may benefit from aflibercept more than other patients. ${ }^{43}$

\section{Regorafenib: an oral multikinase inhibitor for the refractory $m C R C$ setting}

Regorafenib, an oral multikinase inhibitor, is an agent recently demonstrated to have efficacy as a single agent in the treatment of refractory mCRC. It inhibits a variety of angiogenic, stromal, and oncogenic kinases, including VEGF receptors (VEGFR)1-3, platelet-derived growth factor receptor- $\beta$ (PDGFR- $\beta$ ), fibroblast growth factor receptor 1 (FGFR1), TIE2, KIT, RET, and $B R A F,{ }^{44}$ thereby distinguishing it from bevacizumab and aflibercept. The Phase III CORRECT trial treated a total of 1,513 mCRC patients refractory to therapy to either placebo or regorafenib $160 \mathrm{mg}$ daily for the first 3 weeks out of every 4-week cycle (Table 1). ${ }^{45}$ The primary endpoint of OS was met at the second interim analysis. Median OS was improved with regorafenib when compared to placebo (6.4 vs 5.0 months, HR: 0.77, 95\% CI: 0.64-0.94, one-sided $P=0.0052)$. Median PFS for regorafenib versus placebo was also improved (1.9 vs 1.7 months, HR: 0.49 , 95\% CI: $0.42-0.58, P<0.0001)$, but ORR were similarly small $(1.0 \%$ vs $0.4 \%, P=0.19)$. Despite the small survival benefit, however, regorafenib treatment led to significant toxicities, with $93 \%$ of patients receiving regorafenib experiencing treatmentrelated adverse events when compared with $61 \%$ of patients receiving placebo. Like aflibercept, predictive biomarkers to predict which patients would most benefit from regorafenib have not yet been discovered.

\section{Future directions for personalized therapy in $\mathrm{MCRC}$}

Despite promising data regarding the use of biologic therapy in $\mathrm{mCRC}$, more knowledge is needed to optimize and refine their use in the appropriate patient settings. Recent advances in the molecular characterization of CRC are building a foundation for further refinement of existing drugs and development of new targeted therapies. For example, in 2012, The Cancer Genome Atlas (TCGA) Network published results from multidimensional genomic analyses of 276 human colorectal carcinomas, the first comprehensive study of its kind. ${ }^{46}$ These analyses provided insights into pathways dysregulated in $\mathrm{CRC}$, and perhaps more importantly, those amenable to therapeutic targeting. As a result, potential targets under preclinical and clinical investigation for the treatment of CRC include those in the Wnt/beta-catenin pathway, the $\mathrm{PI} 3 \mathrm{~K} / \mathrm{Akt} / \mathrm{mTOR}$ pathway, and the TGF- $\beta$ pathway. Analyses such as these from TCGA and others will provide necessary biological infrastructure for the development of more novel and effective therapies for $\mathrm{mCRC}$.

In addition to understanding the biological underpinnings of this complex and often heterogeneous disease, improved knowledge regarding the mechanisms of action of the targeted therapies is needed to employ these most effectively. Once these mechanisms of initial response and intrinsic and acquired resistance are elucidated, development of accurate predictive biomarkers should be possible. As the FIRE-3 and PRIME data demonstrate, even currently established predictive biomarkers such as $K R A S$ exon 2 mutations are not the definitive answer for anti-EGFR therapy selection; furthermore, biomarkers for the anti-VEGF agents are lacking. Availability of robust markers of response based on biological mechanisms of disease and therapy will not only minimize ineffective treatments for mCRC patients, but also improve response rates and survival benefits of established and novel drugs through accurate patient selection and therapy matching.

\section{Conclusion}

mCRC remains a significant cause of cancer-related death worldwide. While advances in drug development over the last 
two decades have expanded the number of therapies at our disposal for this disease, several significant challenges regarding the optimization of these therapies remain. Better understanding of the mechanisms of action of our targeted therapies such as cetuximab, panitumumab, bevacizumab, aflibercept, and regorafenib is needed to determine the most appropriate patients to treat with these agents. Insight into the effects of combining targeted therapies with cytotoxic chemotherapy, as well as the best sequence of agents to employ, is vital. Knowledge of potential resistance mechanisms of targeted agents, both intrinsic and acquired, will also play a key role in the treatment of this complex disease. Finally, development of new agents, predictive biomarkers, and more effective combinatorial therapy will be possible through scientific knowledge gleaned through emerging molecular technologies.

\section{Disclosure}

KKC has served on a scientific advisory board for Bayer. The authors have no other conflicts of interest to report in this work.

\section{References}

1. Siegel R, Naishadham D, Jemal A. Cancer statistics, 2013. CA Cancer J Clin. 2013;63(1):11-30.

2. Lynch JP, Hoops TC. The genetic pathogenesis of colorectal cancer. Hematol Oncol Clin North Am. 2002;16(4):775-810.

3. Fearon ER, Vogelstein B. A genetic model for colorectal tumorigenesis. Cell. 1990;61(5):759-767.

4. Weisenberger DJ, Siegmund KD, Campan M, et al. CpG island methylator phenotype underlies sporadic microsatellite instability and is tightly associated with BRAF mutation in colorectal cancer. Nat Genet. 2006;38(7):787-793.

5. Noffsinger AE. Serrated polyps and colorectal cancer: new pathway to malignancy. Annu Rev Pathol. 2009;4:343-364.

6. Rajagopalan H, Bardelli A, Lengauer C, Kinzler KW, Vogelstein B, Velculescu VE. Tumorigenesis: RAF/RAS oncogenes and mismatchrepair status. Nature. 2002;418(6901):934.

7. Domingo E, Niessen RC, Oliveira C, et al. BRAF-V600E is not involved in the colorectal tumorigenesis of HNPCC in patients with functional MLH1 and MSH2 genes. Oncogene. 2005;24(24):3995-3998.

8. Van Cutsem E, Köhne CH, Hitre E, et al. Cetuximab and chemotherapy as initial treatment for metastatic colorectal cancer. $N$ Engl J Med. 2009;360(14):1408-1417.

9. Van Cutsem E, Köhne CH, Láng I, et al. Cetuximab plus irinotecan, fluorouracil, and leucovorin as first-line treatment for metastatic colorectal cancer: updated analysis of overall survival according to tumor KRAS and BRAF mutation status. J Clin Oncol. 2011;29(15):2011-2019.

10. Sobrero AF, Maurel J, Fehrenbacher L, et al. EPIC: phase III trial of cetuximab plus irinotecan after fluoropyrimidine and oxaliplatin failure in patients with metastatic colorectal cancer. J Clin Oncol. 2008;26(14): 2311-2319.

11. Bokemeyer C, Bondarenko I, Makhson A, et al. Fluorouracil, leucovorin, and oxaliplatin with and without cetuximab in the first-line treatment of metastatic colorectal cancer. J Clin Oncol. 2009;27(5):663-671.

12. Douillard JY, Siena S, Cassidy J, et al. Randomized, phase III trial of panitumumab with infusional fluorouracil, leucovorin, and oxaliplatin (FOLFOX4) versus FOLFOX4 alone as first-line treatment in patients with previously untreated metastatic colorectal cancer: the PRIME study. J Clin Oncol. 2010;28(31):4697-4705.
13. Douillard JY, Siena S, Tabernero J, et al. Overall survival analysis from PRIME: randomized phase III study of panitumumab (pmab) with FOLFOX4 for first-line metastatic colorectal cancer (mCRC). J Clin Oncol. 2013;31 Suppl: abstr 3620.

14. Peeters M, Price TJ, Cervantes A, et al. Randomized phase III study of panitumumab with fluorouracil, leucovorin, and irinotecan (FOLFIRI) compared with FOLFIRI alone as second-line treatment in patients with metastatic colorectal cancer. J Clin Oncol. 2010;28(31): 4706-4713.

15. Jonker DJ, O'Callaghan CJ, Karapetis CS, et al. Cetuximab for the treatment of colorectal cancer. $N$ Engl J Med. 2007;357(20): 2040-2048.

16. Van Cutsem E, Peeters M, Siena S, et al. Open-label phase III trial of panitumumab plus best supportive care compared with best supportive care alone in patients with chemotherapy-refractory metastatic colorectal cancer. J Clin Oncol. 2007;25(13):1658-1664.

17. Price TJ, Peeters M, Kim TW, et al. ASPECCT: a randomized, multicenter, open-label, phase 3 study of panitumumab (pmab) vs cetuximab (cmab) for previously treated wild-type (WT) KRAS metastatic colorectal cancer (mCRC). Eur J Cancer. 2013;49 Suppl 3: LBA 18.

18. Ye LC, Liu TS, Ren L, et al. Randomized controlled trial of cetuximab plus chemotherapy for patients with KRAS wild-type unresectable colorectal liver-limited metastases. J Clin Oncol. 2013;31(16): 1931-1938.

19. Heinemann V, von Weikersthal LF, Decker T, et al. Randomized comparison of FOLFIRI plus cetuximab versus FOLFIRI plus bevacizumab as first-line treatment of KRAS wild-type metastatic colorectal cancer: German AIO study KRK-0306 (FIRE-3). J Clin Oncol. 2013;31 Suppl: abstr LBA3506.

20. Venook AP, Blanke CD, Niedzwiecki D, et al. Cancer and Leukemia Group B/Southwest Oncology Group Trial 80405: a phase III trial of chemotherapy and biologic agents for patients with untreated advanced colorectal adenocarcinoma. Clin Colorectal Cancer. 2005;5(4): 292-294.

21. Venook AP, Blanke CD, Niedzwiecki D, et al. Revisiting the Cancer and Leukemia Group B/Southwest Oncology Group 80405 trial: a phase III trial of chemotherapy and biologic agents for patients with untreated advanced colorectal adenocarcinoma. Clin Colorectal Cancer. 2007;6(7):536-538.

22. Douillard JY, Oliner KS, Siena S, et al. Panitumumab-FOLFOX4 treatment and RAS mutations in colorectal cancer. $N$ Engl $J$ Med. 2013;369(11):1023-1034.

23. Stintzing S, Jung A, Rossius L, et al. Analysis of KRAS/NRAS and BRAF mutations in FIRE-3: a randomized phase III study of FOLFIRI plus cetuximab or bevacizumab as first-line treatment for wild-type KRAS (exon 2) metastatic colorectal cancer patients. Eur J Cancer. 2013;49 Suppl 3: LBA17.

24. Hurwitz H, Fehrenbacher L, Novotny W, et al. Bevacizumab plus irinotecan, fluorouracil, and leucovorin for metastatic colorectal cancer N Engl J Med. 2004;350(23):2335-2342.

25. Fuchs CS, Marshall J, Mitchell E, et al. Randomized, controlled trial of irinotecan plus infusional, bolus, or oral fluoropyrimidines in firstline treatment of metastatic colorectal cancer: results from the BICC-C Study. J Clin Oncol. 2007;25(30):4779-4786.

26. Hochster HS, Hart LL, Ramanathan RK, et al. Safety and efficacy of oxaliplatin and fluoropyrimidine regimens with or without bevacizumab as first-line treatment of metastatic colorectal cancer: results of the TREE study. J Clin Oncol. 2008;26(21):3523-3529.

27. Saltz LB, Clarke S, Díaz-Rubio E, et al. Bevacizumab in combination with oxaliplatin-based chemotherapy as first-line therapy in metastatic colorectal cancer: a randomized phase III study. J Clin Oncol. 2008; 26(12):2013-2019.

28. Fuchs CS, Marshall J, Barrueco J. Randomized, controlled trial of irinotecan plus infusional, bolus, or oral fluoropyrimidines in first-line treatment of metastatic colorectal cancer: updated results from the BICC-C study. J Clin Oncol. 2008;26(4):689-690. 
29. Giantonio BJ, Catalano PJ, Meropol NJ, et al; Eastern Cooperative Oncology Group Study E3200. Bevacizumab in combination with oxaliplatin, fluorouracil, and leucovorin (FOLFOX4) for previously treated metastatic colorectal cancer: results from the Eastern Cooperative Oncology Group Study E3200. J Clin Oncol. 2007;25(12):1539-1544.

30. Grothey A, Sugrue MM, Purdie DM, et al. Bevacizumab beyond first progression is associated with prolonged overall survival in metastatic colorectal cancer: results from a large observational cohort study (BRiTE). J Clin Oncol. 2008;26(33):5326-5334.

31. Cohn AL, Bekaii-Saab TS, Bendell JC, et al. Clinical outcomes in bevacizumab(BV)-treated patients (pts) with metastatic colorectal cancer (mCRC): Results from ARIES observational cohort study (OCS) and confirmation of BRiTE data on BV beyond progression (BBP). J Clin Oncol. 2010;28(Suppl 15): abstr 3596.

32. Bennouna J, Sastre J, Arnold D, et al; ML18147 Study Investigators. Continuation of bevacizumab after first progression in metastatic colorectal cancer (ML18147): a randomised phase 3 trial. Lancet Oncol. 2013;14(1):29-37.

33. Masi G, Vasile E, Loupakis F, et al. Randomized trial of two induction chemotherapy regimens in metastatic colorectal cancer: an updated analysis. J Natl Cancer Inst. 2011;103(1):21-30.

34. Falcone A, Ricci S, Brunetti I, et al; Gruppo Oncologico Nord Ovest. Phase III trial of infusional fluorouracil, leucovorin, oxaliplatin, and irinotecan (FOLFOXIRI) compared with infusional fluorouracil, leucovorin, and irinotecan (FOLFIRI) as first-line treatment for metastatic colorectal cancer: the Gruppo Oncologico Nord Ovest. J Clin Oncol. 2007;25(13):1670-1676.

35. Souglakos J, Androulakis N, Syrigos K, et al. FOLFOXIRI (folinic acid, 5-fluorouracil, oxaliplatin and irinotecan) vs FOLFIRI (folinic acid, 5-fluorouracil and irinotecan) as first-line treatment in metastatic colorectal cancer: a multicentre randomised phase III trial from the Hellenic Oncology Research Group (HORG). Br J Cancer. 2006;94(6): 798-805.

36. Falcone A, Cremolini C, Masi G, et al. FOLFOXIRI/bevacizumab (bev) versus FOLFIRI/bev as first-line treatment in unresectable metastatic colorectal cancer (mCRC) patients (pts): Results of the phase III TRIBE trial by GONO group. J Clin Oncol. 2013;31 Suppl: abstr 3505.

37. Loupakis F, Cremolini C, Masi G, et al. FOLFOXIRI plus bevacizumab (bev) versus FOLFIRI plus bev as first-line treatment of metastatic colorectal cancer (MCRC): Results of the phase III randomized TRIBE trial. J Clin Oncol. 2012;31 Suppl 4: abstr 336.
38. Koopman M, Simkins LH, Tije AJ, et al. Maintenance treatment with capecitabine and bevacizumab versus observation after induction treatment with chemotherapy and bevacizumab in metastatic colorectal cancer (mCRC): The phase III CAIRO3 study of the Dutch Colorectal Cancer Group. J Clin Oncol. 2013;31 Suppl: abst 3502.

39. Punt CJA, Simkens LHJ, May A, et al. Updated results including quality of life of the phase III CAIRO3 study of the Dutch Colorectal Cancer Group: maintenance treatment with capecitabine and bevacizumab versus observation after induction treatment with chemotherapy and bevacizumab in metastatic colorectal cancer. Eur J Cancer. 2013; 49 Suppl 3: abstr 2166.

40. Johnsson A, Hagman H, Frödin JE, et al. A randomized phase III trial on maintenance treatment with bevacizumab alone or in combination with erlotinib after chemotherapy and bevacizumab in metastatic colorectal cancer: the Nordic ACT trial. Ann Oncol. 2013;24(9):2335-2341.

41. Holash J, Davis S, Papadopoulos N, et al. VEGF-Trap: a VEGF blocker with potent antitumor effects. Proc Natl Acad Sci U S A. 2002;99(17): 11393-11398

42. Van Cutsem E, Tabernero J, Lakomy R, et al. Addition of aflibercept to fluorouracil, leucovorin, and irinotecan improves survival in a phase III randomized trial in patients with metastatic colorectal cancer previously treated with an oxaliplatin-based regimen. J Clin Oncol. 2012;30(28):3499-3506.

43. Tabernero J, Van Cutsem E, Lakomý R, et al. Aflibercept versus placebo in combination with fluorouracil, leucovorin and irinotecan in the treatment of previously treated metastatic colorectal cancer: prespecified subgroup analyses from the VELOUR trial. Eur J Cancer. 2014;50(2): 320-331.

44. Wilhelm SM, Dumas J, Adnane L, et al. Regorafenib (BAY 73-4506): a new oral multikinase inhibitor of angiogenic, stromal and oncogenic receptor tyrosine kinases with potent preclinical antitumor activity. Int J Cancer. 2011;129(1):245-255.

45. Grothey A, Van Cutsem E, Sobrero A, et al; CORRECT Study Group. Regorafenib monotherapy for previously treated metastatic colorectal cancer (CORRECT): an international, multicentre, randomised, placebo-controlled, phase 3 trial. Lancet. 2013;381(9863):303-312.

46. Cancer Genome Atlas Network. Comprehensive molecular characterization of human colon and rectal cancer. Nature. 2012;487(7407): $330-337$.
Pharmacogenomics and Personalized Medicine

\section{Publish your work in this journal}

Pharmacogenomics and Personalized Medicine is an international, peerreviewed, open access journal characterizing the influence of genotype on pharmacology leading to the development of personalized treatment programs and individualized drug selection for improved safety, efficacy and sustainability. This journal is indexed on the American Chemical

\section{Dovepress}

Society's Chemical Abstracts Service (CAS). The manuscript management system is completely online and includes a very quick and fair peer-review system, which is all easy to use. Visit http://www.dovepress. com/testimonials.php to read real quotes from published authors. 